\title{
Validation of a highly integrated SiPM readout system with a TOF-PET demonstrator
}

T. Niknejad, S. Setayeshi, S. Tavernier; R. Bugalho, L. Ferramacho,

A. Di Francesco, C. Leong, M.D. Rolo, M. Shamshirsaz, J.C. Silva, R. Silva, M. Silveira, C. Zorraquino and J. Varela

AвstRact: We have developed a highly integrated, fast and compact readout electronics for Silicon Photomultiplier (SiPM) based Time of Flight Positron Emission Tomography (TOF-PET) scanners. The readout is based on the use of TOP-PET Application Specific Integrated Circuit (PETsys TOFPET1 ASIC) with 64 channels, each with its amplifier, discriminator, Time to Digital Converter (TDC) and amplitude determination using Time Over Threshold (TOT). The ASIC has 25 ps r.m.s. intrinsic time resolution and fully digital output. The system is optimised for high rates, good timing, low power consumption and low cost. For validating the readout electronics, we have built a technical PET scanner, hereafter called "demonstrator", with 2'048 SiPM channels. The PET demonstrator has 16 compact Detector Modules (DM). Each DM has two ASICs reading 128 SiPM pixels in one-to-one coupling to 128 Lutetium Yttrium Orthosilicate (LYSO) crystals measuring $3.1 \times 3.1 \times 15 \mathrm{~mm}^{3}$ each. The data acquisition system for the demonstrator has two Front End Boards type D (FEB/D), each collecting the data of 1'024 channels (8 DMs), and transmitting assembled 
data frames through a serial link ( $4.8 \mathrm{Gbps}$ ), to a single Data Acquisition (DAQ) board plugged into the Peripheral Component Interconnect Express (PCIe) bus of the data acquisition PC. Results obtained with this PET demonstrator are presented.

KeYwords: Gamma camera, SPECT, PET PET/CT, coronary CT angiography (CTA); Instrumentation and methods for time-of-flight (TOF) spectroscopy; Photon detectors for UV, visible and IR photons (solid-state) (PIN diodes, APDs, Si-PMTs, G-APDs, CCDs, EBCCDs, EMCCDs etc); Data processing methods 


\section{Contents}

1 Introduction 1

2 Description of the demonstrator 1

3 Energy resolution $\quad 5$

4 Coincidence time resolution 6

5 Coincidences using the natural radioactivity of lutetium 9

6 Rate capability of the system 11

$\begin{array}{llr}7 & \text { Image reconstruction } & 13\end{array}$

8 Conclusion 15

\section{Introduction}

Many applications require the detection of weak and fast light signals. Until recently, Photomultiplier Tubes (PMT) were universally used for detecting such light signals. In recent years, a new and entirely solid-state light sensor with high gain has become available, the Silicon Photomultiplier (SiPM). SiPMs have now become a cost effective alternative to conventional PMTs in many applications, and in particular for Nuclear Medicine applications. SiPMs are intrinsically faster, are insensitive to magnetic fields and the photosensitive area is easily subdivided in pixels. Additionally they are thin, light and robust, and they only need a few ten volts to operate.

However, for taking full advantage of SiPMs in large systems one needs a fully integrated readout electronics allowing reading each SiPM channel without multiplexing. We have developed such a fast SiPM readout system optimised for Time of Flight Positron Emission Tomography (TOF-PET) applications and scalable to several 10'000 channels. We report on the validation of this readout electronics with a technical test PET scanner, hereafter called "demonstrator", with 2'048 channels.

\section{Description of the demonstrator}

The PET demonstrator ring (figure 1) has an inner diameter of $235 \mathrm{~mm}$ (crystal to crystal distance) and can accommodate 24 Detector Modules (DM). However, for the work reported here the demonstrator was only equipped with 16 DMs forming two arcs of 8 modules facing each other. The DMs are mechanically independent units that fit in the slots of a dedicated aluminium support ring. The mechanical precision of DM position is better than $0.1 \mathrm{~mm}$. 


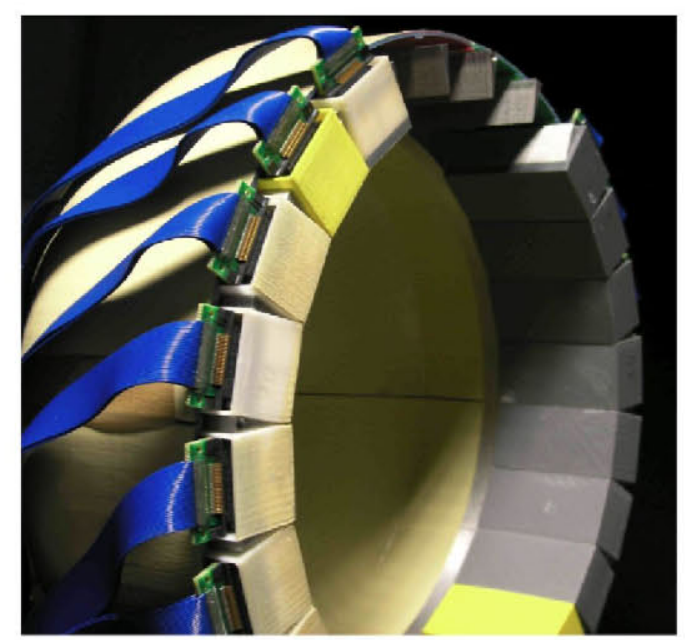

Figure 1. Image of the PET demonstrator ring. The demonstrator has $235 \mathrm{~mm}$ inner diameter, and has two sectors with 8 detector modules each.

Each DM has 8 matrices of $4 \times 4$ Lutetium Yttrium Orthosilicate (LYSO) scintillating crystals, each measuring $3.1 \times 3.1 \times 15 \mathrm{~mm}^{3}$ produced by CPI Inc. ${ }^{1}$ The scintillator pixels are optically separated by $70 \mu \mathrm{m}$ Vikuiti Enhanced Specular Reflector foils. ${ }^{2}$ These scintillator arrays are optically coupled to SiPM arrays from Hamamatsu (S12642-0404PB-50 TSV-MPPC) [1]. The photo-detector pixels have an active area of $3 \times 3 \mathrm{~mm}^{2}$ and are arranged in $4 \times 4$ arrays. Both the LYSO crystal array and the MPPC array have a pitch of $3.2 \mathrm{~mm}$. The crystal matrices and associated MPPCs plug directly in the Front End Boards type A (FEB/A) forming a compact detecting unit with active area $59 \times 29 \mathrm{~mm}^{2}$. The unit, together with a copper cooling plate, is placed in a 3D printed plastic box to form a DM (figure 2). The ring of DMs is placed inside a 3D printed plastic enclosure (figure 1) assuring a laminar flow of cooled air produced by an external cooling unit. The cooled air flows over the copper plates of the DMs and stabilizes the temperature of the DMs to $(19.0 \pm 0.5)^{\circ} \mathrm{C}$.

The readout electronics is based on the TOF-PET Application Specific Integrated Circuit (ASIC) [2, 3]. TOFPET ASIC is a low-noise and low-power 64-channel mixed-mode chip with fully digital output designed in $130 \mathrm{~nm}$ CMOS technology for SiPM readout, developed in the framework of the EndoTOFPET-US project [3]. The ASIC is optimised for timing and has $25 \mathrm{ps}$ time resolution (r.m.s.) [4]. It uses a low threshold for timing and a high threshold for accepting the event. Both thresholds are separately configurable for each channel. Every time one of the 64 channels exceeds the high threshold, an event record is created giving the channel number, the arrival time, and the Time Over Threshold (TOT) of the pulse. Activity in one channel does not cause any dead-time in any of the other channels. There are two Time to Digital Converters (TDC) per channel, one for the arrival time of the pulse and one for determining the TOT. Each of these TDCs consists of four separate and independent Time to Amplitude Converters (TACs). The digitisation time in each TAC is $1.8 \mu$ s on average, but if one TAC is occupied, the next available TAC is used. As a result, event loss due to dead-time is negligible for up to 300 kilo counts per

${ }^{1}$ Crystal Photonics Inc., 5525 Benchmark Lane, Sanford, FL 32773, U.S.A.

${ }^{2} 3 \mathrm{M}$, Vikuiti ${ }^{\mathrm{TM}}$, Enhanced Specular Reflector (ESR). 


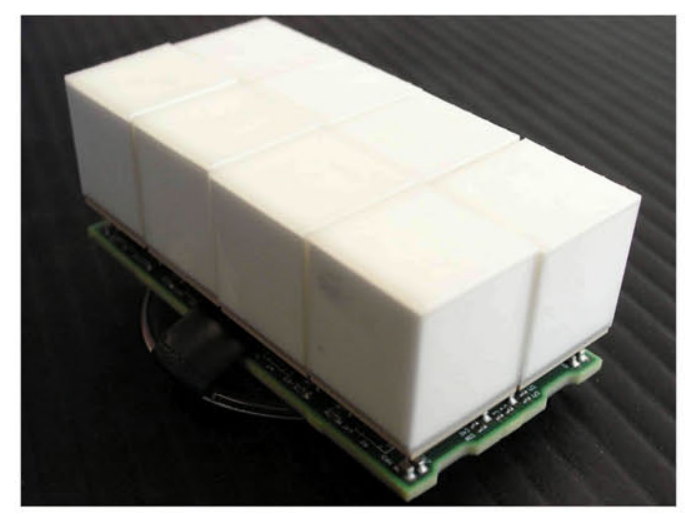

Figure 2. Picture of a Detector Module (DM). It has a FEB/A board with two ASICs, and reads 8 MPPC arrays of 16 pixels each. All MPPC pixels are equipped with a $3.1 \times 3.1 \times 15 \mathrm{~mm}^{3}$ LYSO crystal in one-to-one coupling. In addition, there is an outer plastic box holding it all together, see figure 1.

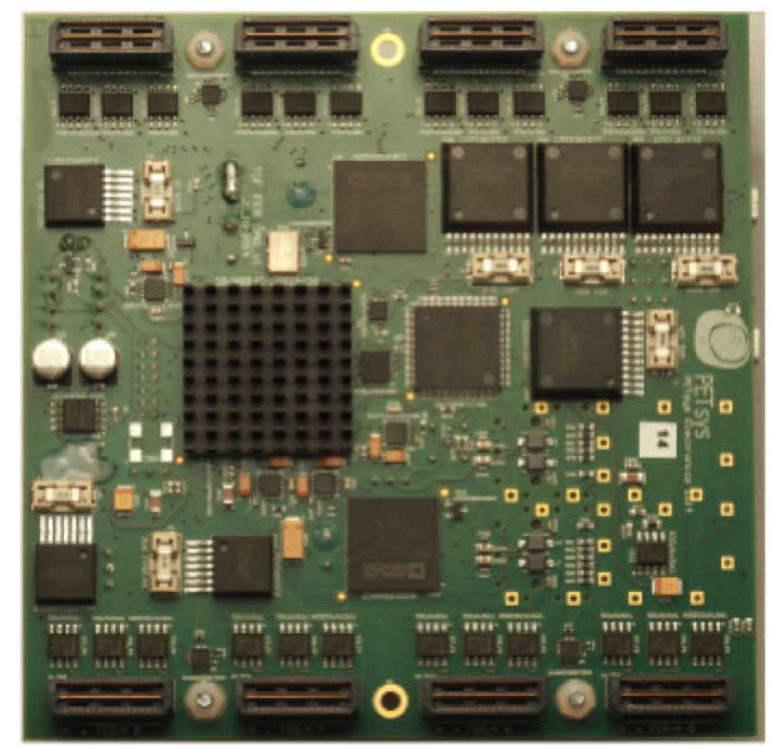

Figure 3. Digital Front End Board type D (FEB/D) used in this study. The board has dimensions $118.0 \times 118.0 \mathrm{~mm}^{2}$.

second (kcps) per channel. Nevertheless, the chip output bandwidth (640 megabits per second (Mbps)) limits the input rate to $100 \mathrm{kcps}$ per channel.

The readout chain is based on the use of three boards [5]. The FEB/A has two ASICs and directly receives the signals from the SiPMs. The Front End Board type D (FEB/D) (figure 3) reads the data from eight FEB/A boards (1'024 channels) and transmits compressed data frames to the Data Acquisition (DAQ) board in the data acquisition PC through a HDMI serial link at 4.8 Gbps. The boards also allow using optical fibers for the communication between the FEB/D and the DAQ, but this possibility was not used in the results reported here.

The FEB/D board has DC-DC converters and regulators providing the required low voltages as well as 64 configurable SiPM bias voltage lines, and distributes clock and reset signals to the ASICs. Several FEB/D boards can be daisy chained, one FEB/D serving as master of several slaves such that one chain is connected to only one DAQ input. 


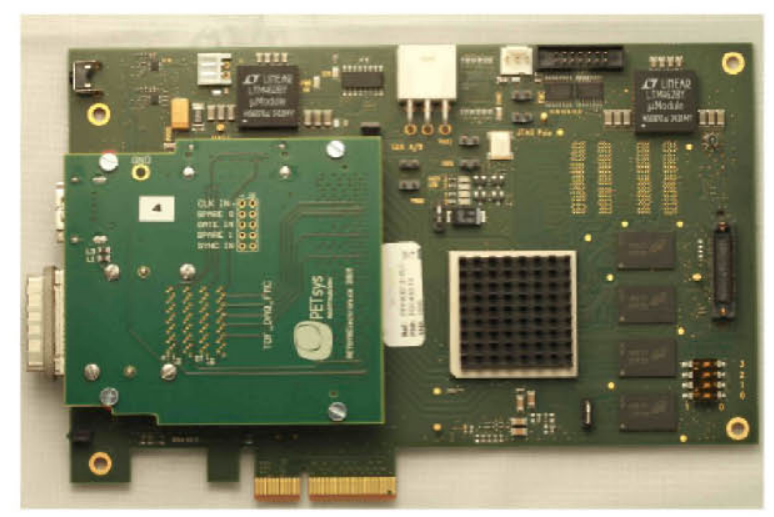

Figure 4. The PCIe Data Acquisition board (DAQ board) used in this study that collects data from the FEB/D boards.

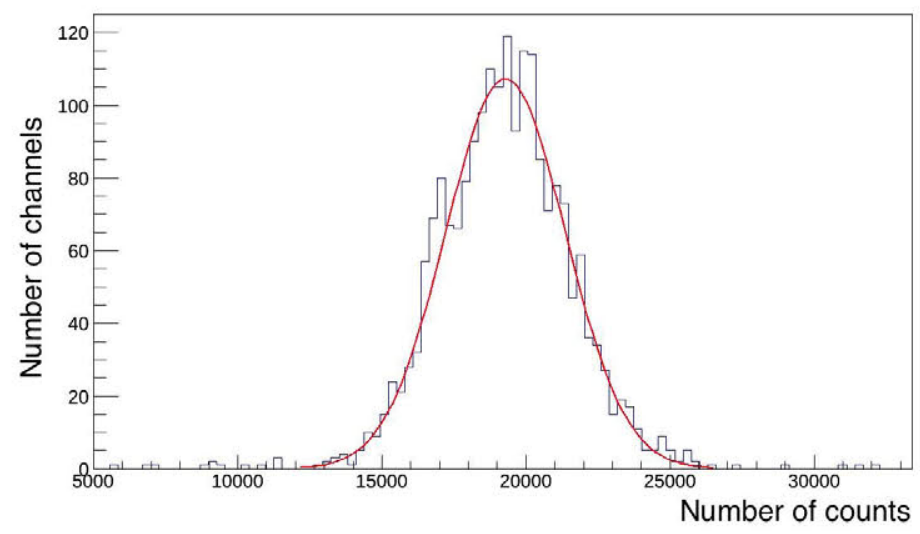

Figure 5. Distribution of counts per channel obtained with ${ }^{68} \mathrm{Ge}$ line source in the centre of the demonstrator along the axis. Events are in the photo-peak ( \pm 1 sigma).

The DAQ board (figure 4) is a Peripheral Component Interconnect Express (PCIe) board that collects data from the FEB/D boards. Up to twelve master FEB/D boards can be connected to the DAQ board, either using HDMI cables, or using optical cables. The DAQ board also provides the system clock $(160 \mathrm{MHz})$ as well as synchronization signals to the FEB/D boards.

The uniformity of the sensitivity of the system is evaluated by placing ${ }^{68} \mathrm{Ge}$ line source in the centre of the demonstrator along the axis. The distribution of the number of counts per channel is shown in figure 5. In this plot, only the events in an interval of \pm 1 sigma around the centre of the $511 \mathrm{keV}$ photo-peak are counted. The dispersion of the sensitivity is calculated as sigma of the distribution and is $10.61 \%$. Except for a few dead channels, the sensitivity is very uniform over the sensitive area.

Figure 6 shows the number of events per channel in the demonstrator when irradiated with a point source of ${ }^{22} \mathrm{Na}$. When the data shown in figure 6 were recorded, the point source was off-centre and a few $\mathrm{cm}$ away from one of the two detector arcs, and only events within the photo-peak $( \pm 1$ sigma) are counted. The two parts in the figure 6 correspond to the two detector arcs. 


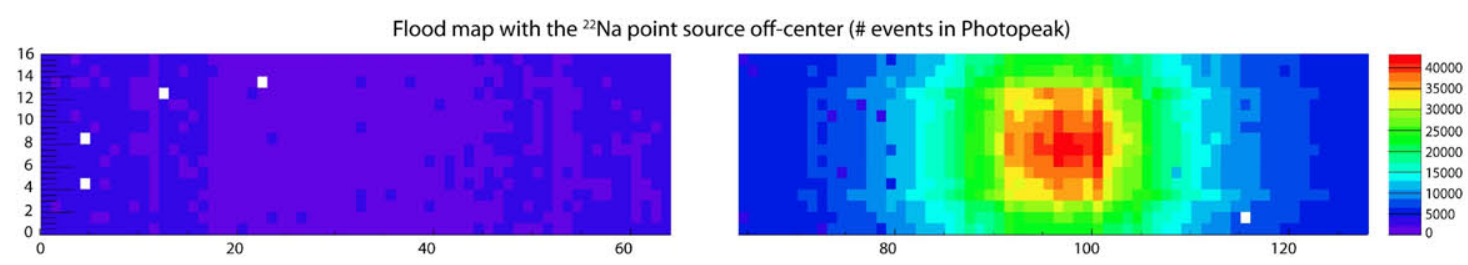

Figure 6. Flood histogram obtained with a ${ }^{22} \mathrm{Na}$ point source close to one of the two detector arcs. This plot shows the number of events in the photo-peak ( \pm 1 sigma) for each pixel. The two parts correspond to the two detector arcs.

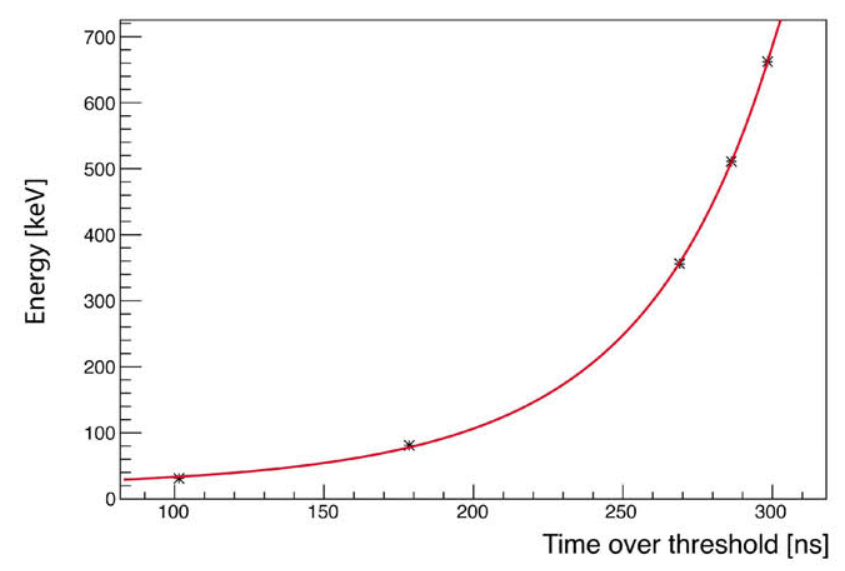

Figure 7. Relation between Time Over Threshold (TOT) and gamma ray energy in one channel.

\section{Energy resolution}

PETSys TOFPET1 ASIC derives the pulse charge from TOT. The relation between TOT and pulse charge is nonlinear, and requires calibration. This can be done using test pulses generated internally in the ASIC with a programmable total charge. These test pulses have a decay time of $40 \mathrm{~ns}$, as is typical for LYSO scintillation signals. However, in the results reported here we use radioactive gamma sources for energy calibration covering the energy range relevant in PET, namely a ${ }^{133} \mathrm{Ba}$ source $(31,81$ and $356 \mathrm{keV})$, a ${ }^{22} \mathrm{Na}$ source $(511 \mathrm{keV})$ and a ${ }^{137} \mathrm{Cs}(662 \mathrm{keV})$. This method of calibration is more accurate than using the internal test pulses because it will also correct the non-linearity of the SiPM response.

The TOT to energy calibration functions (one per channel) can be determined by fitting the data obtained with the radioactive sources, as shown in figure 7 for a particular channel. An exponential function with 3 parameters was used to fit the data with good precision:

$$
\text { Energy }=A \times \exp \left(B \times \mathrm{TOT}^{c}\right)
$$

Figure 8 shows the energy spectrum for all 2'048 channels together after energy calibration. The overall scanner energy resolution is $30 \%$ FWHM at $511 \mathrm{keV}$. The energy resolution for each channel separately is typically $27-28 \%$ FWHM. It should be mentioned that the poor energy resolution is mostly because of saturation of SiPM signal as suggested by figure 7. This is due the fact that the amplifier is almost in saturation for pulses of $511 \mathrm{keV}$. This interpretation is 


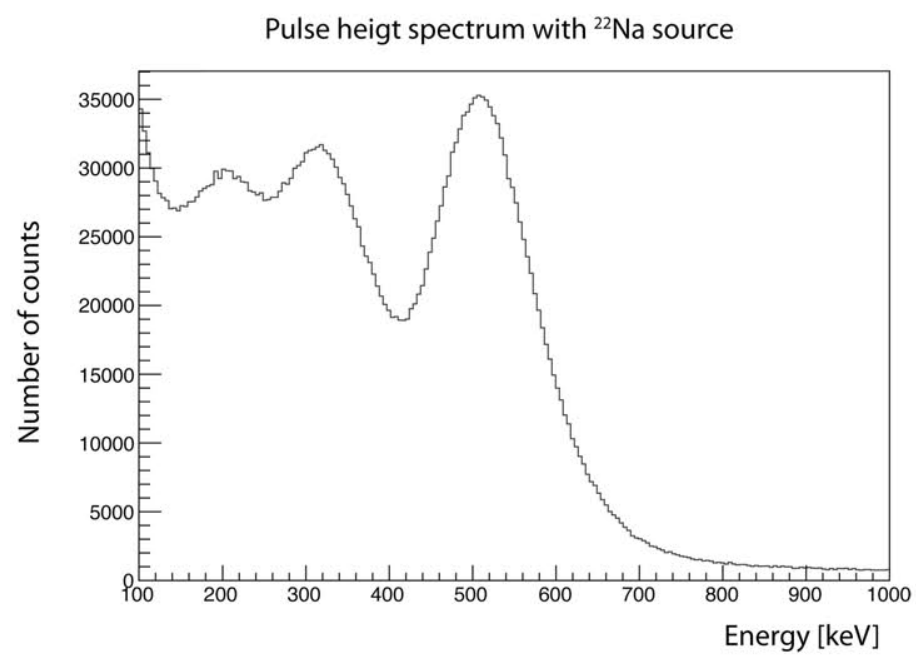

Figure 8. Energy spectrum summed over all channels when irradiating with a ${ }^{22} \mathrm{Na}$ source in the middle of the demonstrator. Only coincidence events between the two halves of the demonstrator were used. The energy resolution is $30 \%$ FWHM.

confirmed by a measurement with a NUV-HD SiPM type from FBK [6]. Only one single crystal with $3.1 \times 3.1 \times 15 \mathrm{~mm}^{3}$ is coupled to one pixel of NUV-HD SiPM with active area of $4 \times 4$ $\mathrm{mm}^{2}$. In this case the pulses have a larger width (TOT $1000 \mathrm{~ns}$ for the $511 \mathrm{keV}$ photo-peak) and have smaller peak amplitude. The energy resolution of $12.5 \%$ FWHM is obtained (see figure 9). Saturation in the output signal can also happen if the number of scintillation photons arriving at the $\mathrm{SiPM}$ is larger than the number of microcells. The $1272 \mathrm{keV}$ peak of ${ }^{22} \mathrm{Na}$ cannot be seen in the energy spectrum obtained by single events with the current electronics and SiPM from Hamamatsu but is seen with the new version of our ASIC. This implies that the saturation effect is not due to the SiPM.

\section{Coincidence time resolution}

The time measurements provided by the TDC are composed by a 10-bit coarse time stamp given by a global clock counter $(160 \mathrm{MHz})$ and a 8-bit fine measurement of the phase of the asynchronous trigger in respect to the global master clock. The fine time measurement uses a set of four Time to Analogue Converters (TAC) and an ADC as described in [2].

The TDCs are calibrated using a procedure that consists of triggering the TDCs with test pulses synchronous to the system clock and varying the test pulse delay in fine steps to obtain the TDC response curve. The test pulses are generated in the field programmable gate arrays (FPGAs) of the FEB/D boards and are distributed to all ASICs in the scanner. The time calibration functions (one per channel) allow correcting for the small non-linearity in the response of the TDCs. Hereafter, this procedure is called TDC calibration.

The measured time stamps for two $511 \mathrm{keV}$ gamma interactions occurring exactly at the same moment are slightly different. This time difference is partly due to a systematic error in the time measured by a given channel, and partly due to a statistical fluctuation on the time measurement. The systematic difference is due to channel-to-channel differences in the clock distribution and 


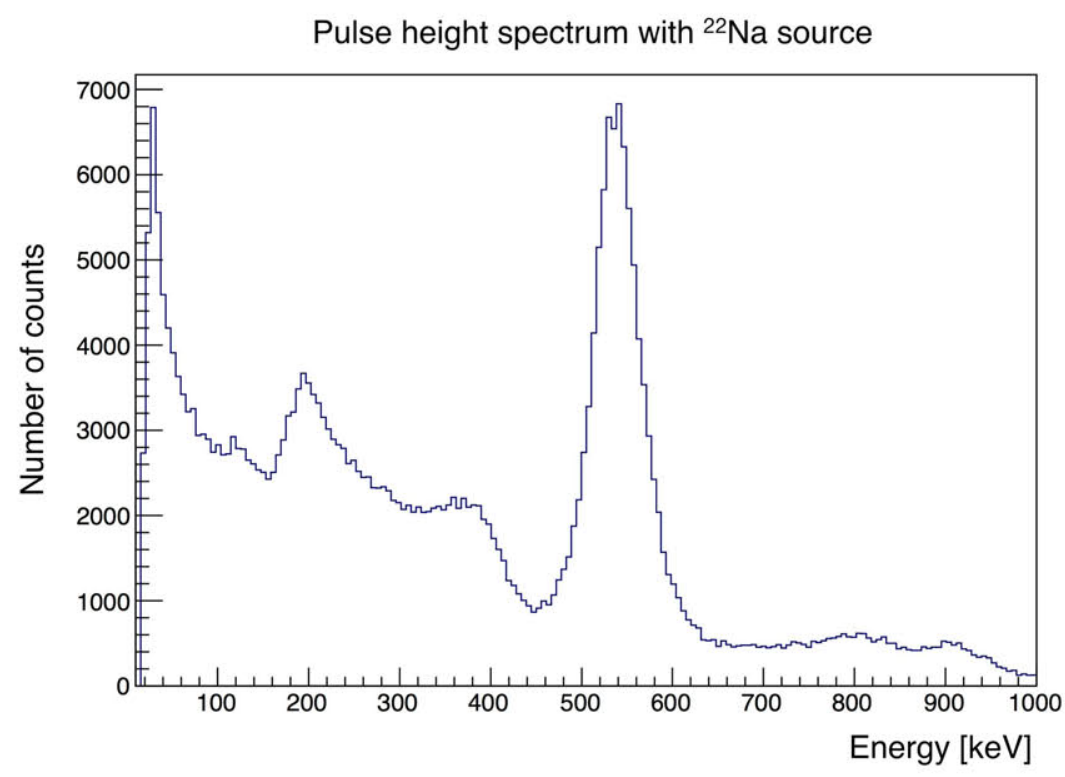

Figure 9. Energy spectrum of the single events in one channel measured with NUV-HD SiPM type from FBK. The energy resolution is $12.5 \%$ FWHM.

can be removed by a time alignment procedure that determines for each channel a time correction hereafter called "time offset". This time offset can be understood as a difference between the average event time measured in one particular channel, compared to the average event time measured by all channels.

The time alignment is performed with an iterative procedure using a rotating ${ }^{68} \mathrm{Ge}$ line source $6 \mathrm{~cm}$ long. The source rotates around the axis of the scanner at a distance of $92 \mathrm{~mm}$. In a sample of coincidence events obtained with this rotating line source we select events where the energy is within \pm 1 sigma of the centre of the $511 \mathrm{keV}$ photo-peak. For each channel $\mathrm{A}$, we take the coincidences which include channel $\mathrm{A}$ and compute the distribution of $\left(t_{\mathrm{A}}-t_{\mathrm{B}}-T O F_{1}\right) \cup\left(t_{\mathrm{A}}-t_{\mathrm{B}}-T O F_{2}\right)$, where $t_{A}$ and $t_{B}$ are the time of arrival in channel $A$ and $B$ respectively and $\mathrm{TOF}_{1}$ and $\mathrm{TOF}_{2}$ are the time of flight difference corresponding to the two possible intersections of LOR_AB with the source's path. Thus, this distribution will contain $50 \%$ of cases with correct TOF, which cluster in a central peak, and $50 \%$ of cases with incorrect TOF which cluster in two lateral peaks. In order to make the peaks identification easier, we restrict the coincidences to the cases where the LOR_AB is at an angle of 5 degrees to the centre of the detector, for which the difference between $\mathrm{TOF}_{1}$ and $\mathrm{TOF}_{2}$ is larger. We then assign a time correction to the detector pixel such that the resulting peak is centred at zero. The process needs to be repeated typically between 5 and 10 times for the time offsets for all 2'048 channels to reach a stable value. Figure 10 shows the distribution of all the time offsets after TDC calibration but before time alignment.

The timing performance of the scanner after the TDC calibration and time alignment is measured with a ${ }^{22} \mathrm{Na}$ point source placed in the geometrical centre of the demonstrator. Figure 11 shows the distribution of coincidence time resolution (FWHM) and the distribution of the mean of the measured time difference for a sample of crystal pixel pairs corresponding to Lines of Response (LOR) going through the point source. The average time difference has a r.m.s. of $20 \mathrm{ps,} \mathrm{and}$ 


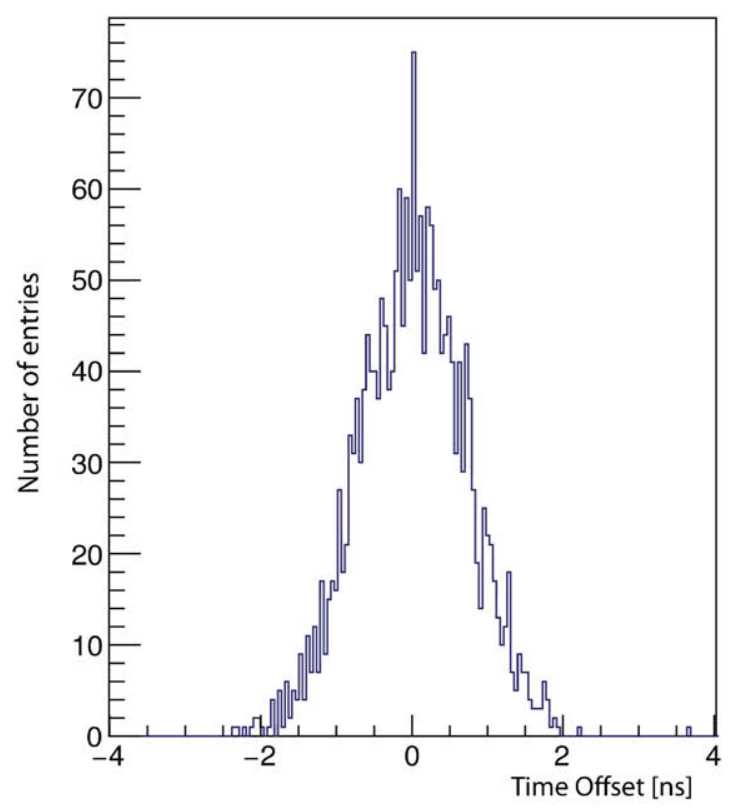

Figure 10. Distribution of the deviation from zero for all time differences in channel pairs after TDC calibration and before time alignment.
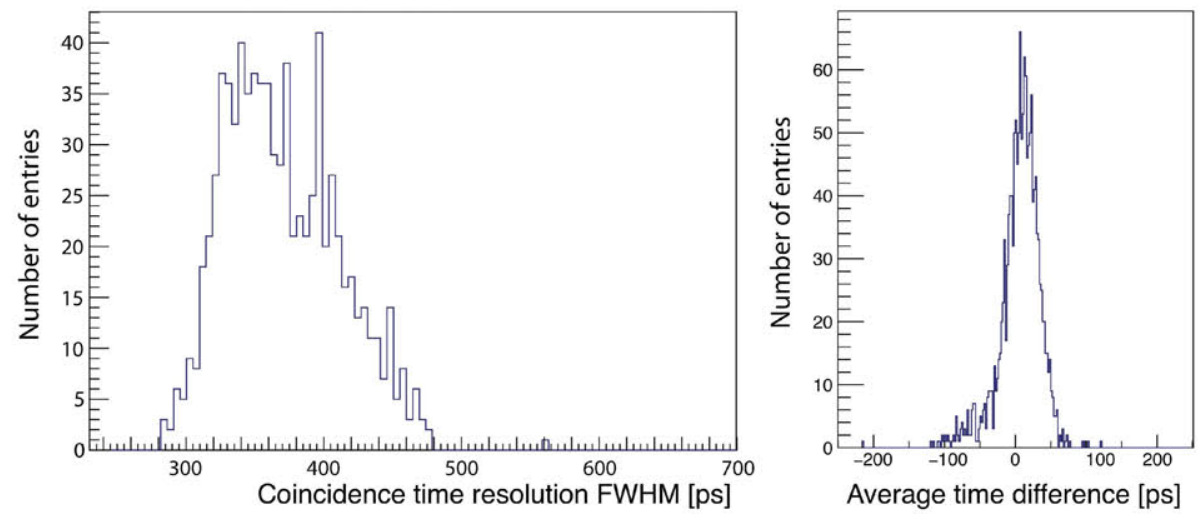

Figure 11. Distribution of the coincidence time resolution (FWHM) (left) and the distribution of the mean of the time difference (right) for a sample of crystal pixel pairs corresponding to Lines of Response (LOR) going through a point source placed in the centre of the scanner.

therefore the uncertainty on the channel time offsets after TDC calibration and time alignment is about 14 ps r.m.s. The average of the distribution of coincidence time resolutions in figure 11 is compatible with the width of the distribution of time differences for all LOR shown in figure 12. The resulting coincidence time resolution is 375 ps FWHM.

The timing resolution with NUV-HD SiPM was also measured by putting tow single crystals face-to-face in coincidence. The resulting coincidence time resolution is $290.7 \mathrm{ps}$ FWHM (see figure 13). The improved coincidence time resolution with NUV-HD SiPM compared to SiPM from Hamamatsu is due to the gain. Higher gain give better signal to noise ratio and hence better time resolution. NUV-HD SiPM gain $\left(3.5 \times 10^{6}\right)$ is about three times higher than Hamamatsu SiPM $\left(1.25 \times 10^{6}\right)$. The gains of Hamamatsu and FBK SiPMs are those quoted by the producers at the recommended overvoltage. 




Figure 12. Coincidence time resolution for all LORs going through a ${ }^{22} \mathrm{Na}$ point source.

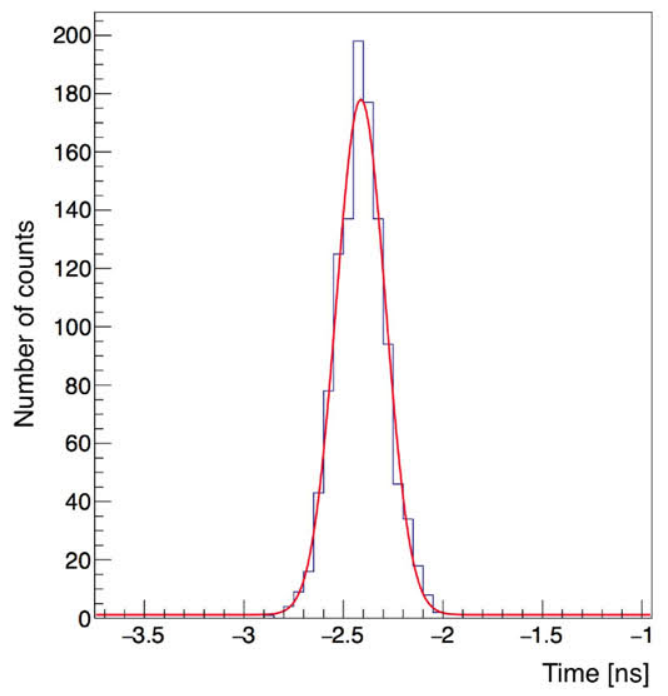

Figure 13. Coincidence time resolution obtained by putting two single crystals in coincidence with NUV-HD SiPM type from FBK. The coincidence time resolution is $290.7 \mathrm{ps}$ FWHM.

\section{Coincidences using the natural radioactivity of lutetium}

To evaluate the performance of the energy calibration method we recorded coincidence events using the natural radioactivity of lutetium. All naturally occurring lutetium contains $2.59 \%$ of ${ }^{176} \mathrm{Lu}$. This isotope decays by beta decay to ${ }^{176} \mathrm{Hf}$ with a decay time of $3.76 \times 10^{10}$ years [7]. As a result, LYSO scintillator is weakly radioactive with 260 decays per $\mathrm{cm}^{3}$ and per second. The beta decay has the end point energy of $593 \mathrm{keV}$, and results $99.61 \%$ of the time in a ${ }^{176} \mathrm{Hf}$ nucleus in the excited state with spin parity $6+$. The excited ${ }^{176} \mathrm{Hf}$ subsequently decays in three steps to the ground state of hafnium. These transitions either go by gamma emission or by electron conversion. The three transitions are listed in the table 1. 
Table 1. Probabilities for gamma emission in the beta decay of ${ }^{176} \mathrm{Lu}$.

\begin{tabular}{|c|c|c|}
\hline Transition & Gamma energy & Gamma emission probability \\
\hline $6+->4+$ & $306.8 \mathrm{keV}$ & $93.6 \%$ \\
\hline $4+>2+$ & $201.8 \mathrm{keV}$ & $78.0 \%$ \\
\hline $2+->0+$ & $88.38 \mathrm{keV}$ & $14.5 \%$ \\
\hline
\end{tabular}

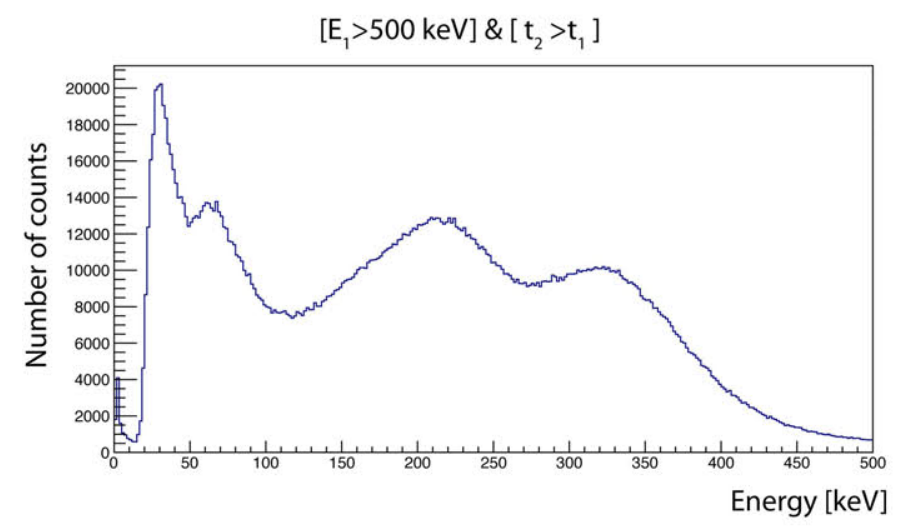

Figure 14. Pulse height spectrum for interactions in one particular pixel of detector 2 , in coincidence with all pixels in the other half of the demonstrator. The event selection is as indicated on the figure.

In addition, in the decay of ${ }^{176} \mathrm{Lu}$ several K-shell X-rays with energy around $60 \mathrm{keV}$ can be emitted.

The emitted beta particles mostly deposit energies in the same crystal array due to the short range. While, gamma rays can be detected not only in the same array, but also in other arrays by escaping outside the first array. An escaped gamma particle detected by another array can make a true intrinsic coincidence event with the first crystal. Therefore, in the absence of any external radioactive source one observes coincidences between the two detector halves due to the natural radioactivity of lutetium. The time difference between the two interactions in such coincidences has two peaks separated by $1.67 \mathrm{~ns}$. These two peaks correspond to events where the lutetium decay took place in one or the other of the two detectors.

Figure 14 shows a typical pulse height spectrum for the interactions in one half of the demonstrator (detector 2), in coincidence with all pixels of the other half of the demonstrator (detector 1). Events were selected by requiring that the energy in detector 1 is larger than $500 \mathrm{keV}$ (the emitting crystal containing a beta particle and one or more gamma rays), and that the time $t_{2}$ is larger than $t_{1}$ to only obtain the events where the the decay happens in detector 1 (a beta particle and one or more gamma rays) and the escaped gamma is absorbed in detector 2. Figure 14, the energy spectrum in detector 2, clearly shows the peaks corresponding to the two gamma rays at $306.8 \mathrm{keV}$ and $201.8 \mathrm{keV}$. The Compton edge of the 307 peak is at $180 \mathrm{keV}$. As a result the peak at $202 \mathrm{keV}$ looks larger than the peak at $307 \mathrm{keV}$. The peak around $65 \mathrm{keV}$ is most likely due to a combination of X-rays, the $88 \mathrm{keV}$ gamma ray, and the Compton edge of the $202 \mathrm{keV}$ peak at $90 \mathrm{keV}$.

We observe that the peak at $202 \mathrm{keV}$ is not exactly at the right position and shows a deviation of about $10-15 \mathrm{keV}$. This can be caused by the energy calibration being slightly wrong in this energy 


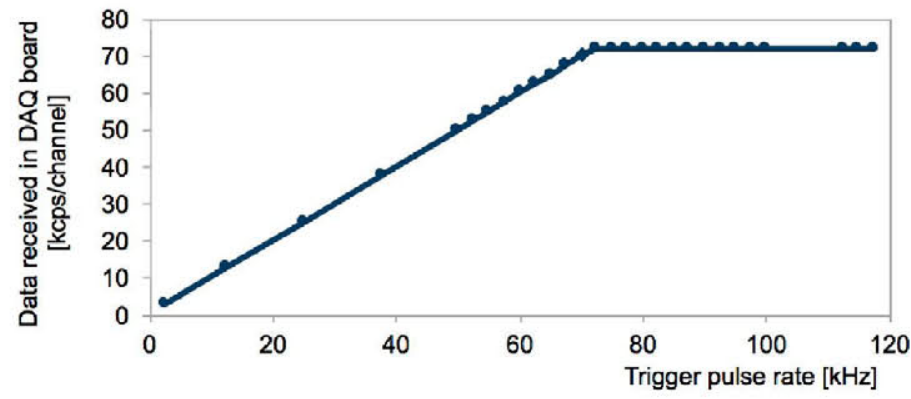

Figure 15. Data output rate of a FEB/D board fully equipped with eight FEB/A boards. For this test we used the internal test pulse generator of the ASIC.

range. This is not surprising since we didn't use any calibration gamma rays with energy between $81 \mathrm{keV}$ and $356 \mathrm{keV}$.

\section{Rate capability of the system}

The total event rate the ASIC can handle is limited by the bandwidth of digital output links. The ASIC has two data output links, each transferring data at $160 \mathrm{MHz}$, two bits per cycle (DDR mode). The total output rate of the ASIC therefore is $640 \mathrm{Mbps}$. In the ASIC each event record has 100 bits, and the total count rate of the ASIC is therefore limited to $6.4 \mathrm{Mcps}$, or $100 \mathrm{kcps}$ per channel. If the event rate is larger than $100 \mathrm{kcps}$ per channel, the system will be losing frames, and the data rate will be severely affected.

In the setup version used in this study, the data acquisition rate of the readout system is limited by the link between the FEB/D and the DAQ boards. Indeed, the connection between the FEB/D board and the DAQ boards uses an HDMI cable transferring at 4.8 Gbps. The event records are compressed to 66 bits in the firmware of the FEB/D. Therefore total data transfer rate from the FEB/D board to the DAQ board is limited to $72 \mathrm{kcps}$ per channel if one FEB/D is fully equipped with $8 \mathrm{FEB} / \mathrm{A}$ boards. To verify the rate limitations discussed above, we made use of the periodic test pulses that can be generated in the ASIC at a controllable rate. All the channels are triggered at the same rate. Since the events are organised in frames and stored in FIFO memories before being transmitted the rate performance of the data acquisition system is not affected by the random nature of the radiation events.

Figure 15 was obtained with one FEB/D board equipped with eight FEB/A. In this case there are 1'024 channels connected to one FEB/D, and we observe that the rate to the DAQ board is limited to $72 \mathrm{kcps}$ per channel, in agreement with expectations.

To test the rate capability of the ASIC itself, we equipped one FEB/D with only four FEB/A. In this case there are only 512 channels connected to one FEB/D and the data transfer rate to the DAQ boards is not limited by the link between the FEB/D and the DAQ boards, and we can see the limitation of the ASIC itself. In figure 16 we see that there is negligible data loss for a trigger rate up to $100 \mathrm{kHz}$. Above $100 \mathrm{kHz}$ the loss becomes important.

It should also be mentioned that the data transfer rate to the computer is limited to 2'000 $\mathrm{MB} / \mathrm{s}$, which corresponds to $240 \mathrm{Mcps}$ for the complete system. The present system only has 2'048 channels; therefore we do not suffer from this limitation. However, for larger systems this 


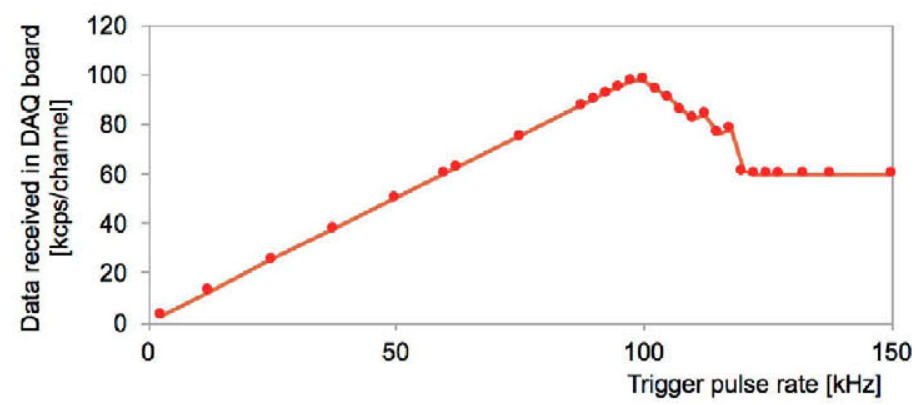

Figure 16. Data output rate of the ASIC as measured with the internal test pulse generator of the ASIC. For this test we used a FEB/D boards equipped with only four FEB/A boards.

will possibly be a bottleneck. The total event rate received from all FEB/D boards can significantly exceed the above transfer rate to the computer. Therefore, a configurable coincidence filter is implemented in the DAQ_V2 firmware.

The coincidence trigger is based on the coarse time and TOT information. It has a resolution of 1 clock cycle $(6.25 \mathrm{~ns})$. The scanner is subdivided in up to maximum 12 trigger sectors, and only coincidences between events in different trigger sectors are considered. All detector modules in each trigger sector are connected to one of the twelve input channels in the DAQ. The list of valid trigger sector combinations is configurable.

The default mode is as follows.

- The trigger algorithm searches the event streams for events having a TOT larger than some minimum TOT.

- A coincidence between two sectors is accepted if two events are found with a time difference of less than 2 clock cycles (coincidence window).

- If a valid coincidence is found, all events in the full scanner in a window beginning 1 clock cycle before and ending 16 clock cycles after the trigger events, are kept and transmitted to the computer (1/16 pre-aft samples).

As an example, we divided the demonstrator into four regions and accepted the coincidence events between each two regions. Using the following parameters for the coincidence trigger, minimum TOT of $112.5 \mathrm{~ns}$ (corresponds to $\sim 50 \mathrm{keV}$ ), coincidence window of $25 \mathrm{~ns}$ and $1 / 16$ pre-aft samples we saw $79.39 \%$ reduction in data output rate.

There are two common methods for removing random events: 1) estimating the random event rate from measurements of the single event rates, or 2) employing a delayed coincidence timing window [8]. Based on these two methods, two procedures are foreseen to collect data for random correction.

- Periodic single trigger: in addition to coincidences, we can measure the single event rates. If the count rate is below the system limit, we count them all. At high count rate, to avoid saturation in any parts of the electronic system, we can sample the data by selecting the events in a window of $10,20,50$ or 100 clock periods every 1025 clock periods. The 1025 clock periodicity ensures the data collected is de-correlated from the system's 1024 clock frame period. 
- Wide coincidence window: the coincidence window can be set to a value larger than 2 clock cycles, allowing the collection of known random events; this is broadly equivalent to the delayed coincidence timing window method.

The rate reduction obtained with the online coincidence filter will depend on the geometry of the scanner and on the settings chosen. With the demonstrator, considering two trigger sectors, we observed a rate reduction of about an order of magnitude with the coincidence trigger.

\section{Image reconstruction}

To show that the data acquired with the demonstrator are correct, we used a point source and a line source to evaluate the system performance and image quality. 3D images are reconstructed using the maximum likelihood expectation maximization (ML-EM) algorithm in open access Software for Tomographic Image Reconstruction (STIR) [9].

To correct for the variation in detection efficiency between different LORs, usually called normalisation, we used a rotating ${ }^{68} \mathrm{Ge}$ line source that is slightly longer than the axial field of view. The line source is parallel to the axis of the scanner and rotating at a distance of $92 \mathrm{~mm}$ around the axis of the scanner. We use a maximum likelihood based estimate of normalisation factors as in [10]. In this work we don't need to correct for the attenuation.

$\mathrm{A}^{22} \mathrm{Na}$ point source was placed very close to the centre in axial direction, while in the transaxial plane it was approximately $1 \mathrm{~cm}$ off-centre. By drawing profiles in the reconstructed image, the Gaussian fits gave 2.40, 3.06 and 2.41 mm FWHM in the X, Y and Z direction respectively. The degradation in $\mathrm{Y}$ direction is due the incomplete angular coverage. No correction was applied for the dimension of the source. Figure 17 shows the orientation of the axes. In figure 18, the image of a point source and the corresponding profile fit results are shown.

Similarly, the image of a $1.5 \mathrm{~mm}$ diameter ${ }^{68} \mathrm{Ge}$ linear source placed in the centre along the axis of the scanner is reconstructed and an average of $3.2 \mathrm{~mm}$ FWHM was measured in trans-axial plane (figure 19).

The spatial resolution of the system was evaluated using NEMA NU 4-2008 procedure in a full ring geometry [11]. Since the demonstrator is not yet mechanically prepared to be rotated to have a complete angular coverage, simulation of the full ring demonstrator were performed using the GEANT4 Application in Tomographic Emission (GATE) Monte Carlo simulation package [12]. The ${ }^{22} \mathrm{Na}$ point source is placed in the centre of the axial field of view (FOV) and at the radial distance of $10 \mathrm{~mm}$ from the centre in air. The image of the point source is reconstructed using the 3D Reprojection algorithm in STIR (FBP-3DRP). The average spatial resolution over transaxial and axial directions is $3.50 \mathrm{~mm}$ FWHM. To validate the obtained result from the simulation data with the real data from the scanner, the demonstrator with the current partial ring geometry was simulated and the image of the ${ }^{22} \mathrm{Na}$ point source $10 \mathrm{~mm}$ off-centre was reconstructed with FBP-3DRP. Gaussian fit of the profiles in X direction (where we have angular coverage) gave 3.14 mm FWHM for simulation data which matches completely the value obtained with the real data (3.28 mm FWHM). 


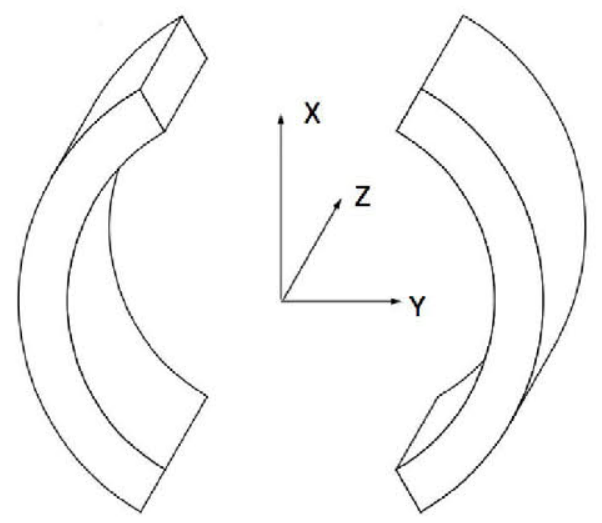

Figure 17. Schematic of the demonstrator showing the axes orientation.

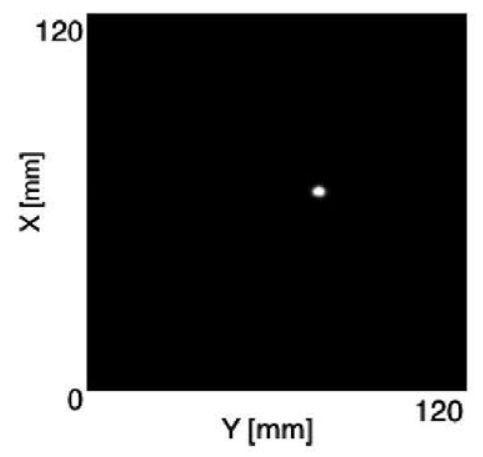

(a)

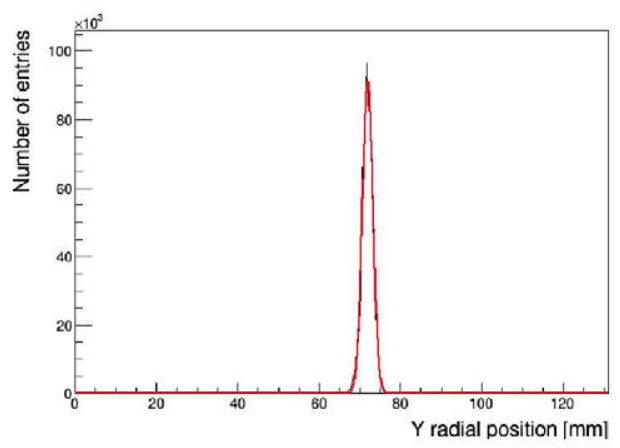

(c)

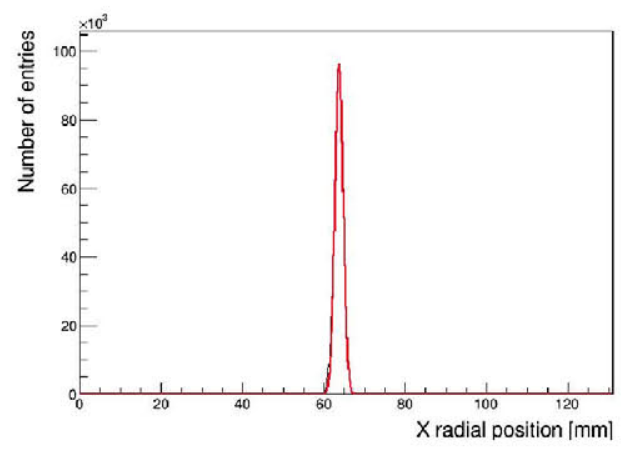

(b)

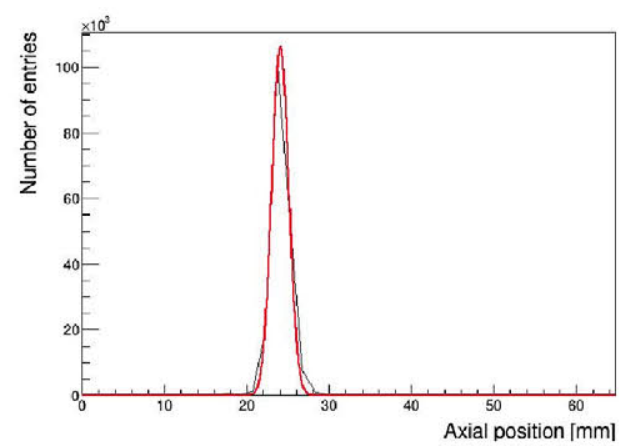

(d)

Figure 18. (a) Image of the central transverse slice for a point source, $1 \mathrm{~cm}$ off-center, and the corresponding profiles along (b) X, (c) Y and (d) Z directions. The measured FWHM resolutions are 2.40, 3.06 and 2.41 $\mathrm{mm}$ respectively. The centre of the scanner in the $X-Y$ plane is at $X=60 \mathrm{~mm}$ and $Y=60 \mathrm{~mm}$. 

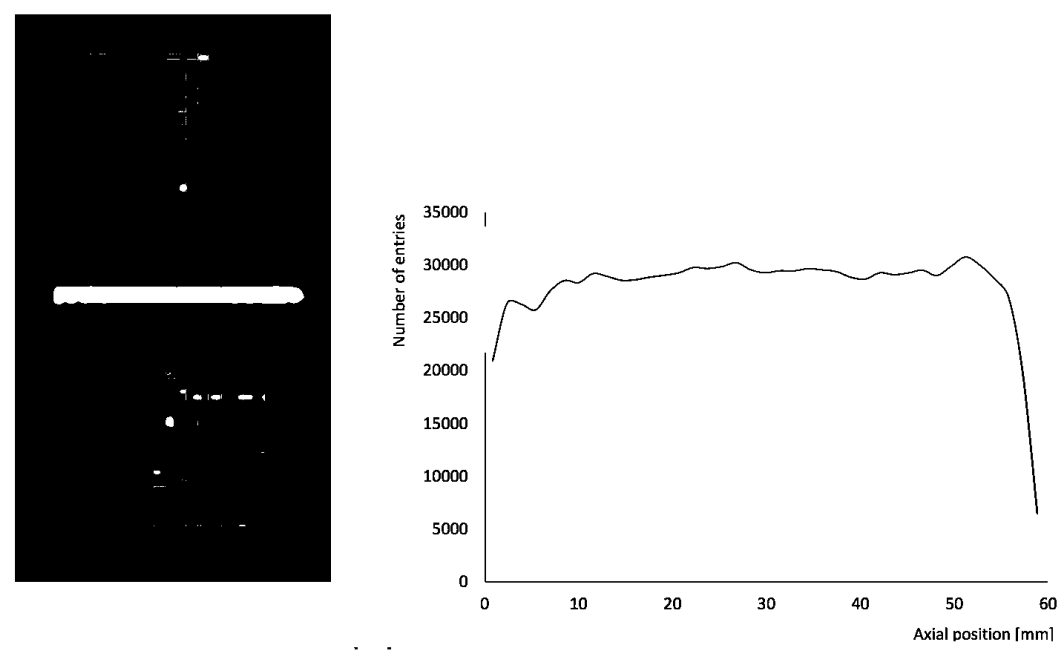

Figure 19. Image of a line source aligned with the axis in the centre of the demonstrator (left) and the longitudinal profile (right).

\section{Conclusion}

We have validated the performance of our readout electronics developed for SiPM based PET scanners. For this validation we used a technical prototype PET scanner with 2'048 channels. Each channel has a $3.1 \times 3.1 \times 15 \mathrm{~mm}^{3}$ LYSO crystal in one-to-one coupling to a SiPM (TSV-MPPC) with pixel size of $3 \times 3 \mathrm{~mm}^{2}$. The SiPM signal readout and digitization uses an ASIC with 64 channels, implementing signal amplification and discrimination, time-to-digital conversion and amplitude determination, featuring fully digital output. The data acquisition chain uses two FEB/D boards, each collecting the data of 1'024 channels, and transmitting assembled data frames through 4.8 Gbps serial links to a single DAQ board plugged into the PCIe bus of the data acquisition PC.

We have shown that the ASIC accepts input SiPM signals at a rate up to $100 \mathrm{kcps}$ without data loss. The total data transfer rate from the FEB/D board to the DAQ board is limited to $72 \mathrm{kcps}$ per channel if one FEB/D is fully equipped with $8 \mathrm{FEB} / \mathrm{A}$ boards. The maximum event rate is expected to become about twice as large by using optical links in a future implementation.

The data obtained with a ${ }^{68} \mathrm{Ge}$ radioactive line source showed a sensitivity that is homogeneous over the scanner. The dispersion of the sensitivity is calculated as $10.61 \%$ sigma. The average coincidence time resolution of the full PET demonstrator after TDC calibration and time alignment is 375 ps FWHM. The energy resolution is obtained with TOT measurement is found to be $30 \%$ FWHM at $511 \mathrm{keV}$ events. This poor energy resolution is mostly due to a mismatch between the TSV-MPPC from Hamamatsu and the ASIC characteristics, implying that the amplifier is almost in saturation for pulses of $511 \mathrm{keV}$. A measurement with a NUV-HD SiPM type from FBK having higher gain than TSV-MPPC and yielding pulses with larger width and smaller peak amplitude showed an energy resolution of $12.5 \%$ FWHM and a coincidence time resolution of $290.7 \mathrm{ps}$.

To verify that the data obtained with the demonstrator is correct, the image reconstruction was performed using the ML-EM algorithm in the open access STIR software with the acquired data from point and linear sources. The spatial resolution of the system is evaluated in a full ring geometry with simulation in GATE and is found to be $3.50 \mathrm{~mm}$ FWHM.

A new version of the ASIC is now under test (PETsys TOFPET2 ASIC) [13]. The new ASIC will have proper charge integration for determining the energy of the event, and this will result in 
an improved energy resolution. The new ASIC will also have improved time resolution and will accept data at $600 \mathrm{kcps}$ per channel. The readout boards FEB/D and DAQ are compatible with the new ASIC.

\section{Acknowledgments}

This work has partly been carried out in the frame of the Crystal Clear Collaboration, under the Contract Agreement KM2912/KT/PH/004C, and was partially supported by a Marie-Curie Early Initial Training Network Fellowship of the European Community's 7th Framework Program under Grant Agreement (PITN-GA-2011-289355- PicoSEC-MCNet). LIP, Lisbon, Portugal; PETsys Electronics, Lisbon, Portugal; Vrije Universiteit Brussel, Belgium, are part of the of the COST action TD 1401, Fast Advanced Scintillation Timing (2014-2018).

\section{References}

[1] Hamamatsu Optical Sensors, http://www.hamamatsu.com/eu/en/product/category/3100/index.html, (Sept., 2015).

[2] M.D. Rolo et al., TOFPET ASIC for PET applications, 2013 JINST 8 C02050.

[3] N. Aubry et al., EndoTOFPET-US: a novel multimodal tool for endoscopy and positron emission tomography, 2013 JINST 8 C04002.

[4] R. Bugalho et al., Design and performance of an ASIC for TOF applications, IEEE Nucl. Sci. Symp. Rec. (2013) 1.

[5] http://www.petsyselectronics.com/web/.

[6] C. Piemonte et al., Performance of NUV-HD silicon photomultiplier technology, IEEE Trans. Electron Dev. 8 (2016) 1111.

[7] National Nuclear data center, Brookhaven national laboratory, http://www.nndc.bnl.gov/.

[8] D. L. Bailey et al., Positron emission tomography: basic science, Springer, Germany (2005).

[9] K. Thielemans et al., STIR: Software for Tomographic Image Reconstruction release 2, Phys. Med. Biol. 57 (2012) 867.

[10] D. Hogg et al., Maximum-likelihood estimation of normalisation factors for PET, IEEE Nucl. Sci. Symp. Rec. 57 (2011) 2065.

[11] Performance Measurements of Small Animal Positron Emission Tomographs. National Electrical Manufacturers Association, NEMA Standards Publication NU 4-2008. Rosslyn, VA, U.S.A. (2008).

[12] S. Jan et al., GATE: a simulation toolkit for PET and SPECT, Phys. Med. Biol. 49 (2004) 4543.

[13] A. Di Francesco et al., TOFPET2: a high-performance ASIC for time and amplitude measurements of SiPM signals in time-of-fight applications, 2016 JINST 63 C03042. 